\title{
Measurements of neutron-induced reactions in inverse kinematics and appli- cations to nuclear astrophysics
}

\author{
René Reifarth ${ }^{1, a}$, Yuri A. Litvinov ${ }^{2}$, Anne Endres ${ }^{1}$, Kathrin Göbel ${ }^{1}$, Tanja Heftrich ${ }^{1}$, Jan Glorius ${ }^{1,2}$, Alexander \\ Koloczek $^{1,2}$, Kerstin Sonnabend ${ }^{1}$, Claudia Travaglio ${ }^{3,4}$, and Mario Weigand ${ }^{1}$ \\ ${ }^{1}$ Goethe University Frankfurt am Main, Frankfurt, Germany \\ ${ }^{2}$ GSI Helmholtzzentrum für Schwerionenforschung, Darmstadt, Germany \\ ${ }^{3}$ INAF-Astronomical Observatory Turin, Turin, Italy \\ ${ }^{4}$ B2FH Association, Turin, Italy
}

\begin{abstract}
Neutron capture cross sections of unstable isotopes are important for neutron-induced nucleosynthesis as well as for technological applications. A combination of a radioactive beam facility, an ion storage ring and a high flux reactor would allow a direct measurement of neutron induced reactions over a wide energy range on isotopes with half lives down to minutes. The idea is to measure neutron-induced reactions on radioactive ions in inverse kinematics. This means, the radioactive ions will pass through a neutron target. In order to efficiently use the rare nuclides as well as to enhance the luminosity, the exotic nuclides can be stored in an ion storage ring. The neutron target can be the core of a research reactor, where one of the central fuel elements is replaced by the evacuated beam pipe of the storage ring. Using particle detectors and Schottky spectroscopy, most of the important neutron-induced reactions, such as $(\mathrm{n}, \gamma),(\mathrm{n}, \mathrm{p}),(\mathrm{n}, \alpha),(\mathrm{n}, 2 \mathrm{n})$, or $(\mathrm{n}, \mathrm{f})$, could be investigated.
\end{abstract}

\section{Introduction}

Almost all of the heavy elements are produced via neutron capture reactions equally shared between $s$ and $r$ process $[1-3]$ and to a very small extent by the $i$ process $[4,5]$. The remaining minor part is produced via photon- and protoninduced reactions during the $p$ process, see Fig. 1. The predictive power of the underlying stellar models is limited because they contain poorly constrained physics components such as convection, rotation or magnetic fields. The crucial link between the observed abundances [6] and the desired parameters of the stellar interiors are nuclear reaction data. In contrast to the $r$ process [7], the isotopes important for the $p$ process [8] as well as for the $s$ and $i$ process [9] are stable or not too far off the valley of stability. The nuclear properties of those nuclei are therefore experimentally much easier to access. While indirect measurements, like the investigation of time-reversed reactions, are very often the only choice because of the short half-lives of the investigated isotopes [10-12], this article is focussing on experimental developments aiming at a better understanding of the $s, i$, and $p$ processes by direct measurements of important neutron capture reactions.

Neutron capture cross sections of stable and unstable isotopes are important for neutron-induced nucleosynthesis [3] as well as for technological applications [13]. Following a review of different astrophysical nucleosynthesis sites with an emphasis on neutron-induced reactions, we

\footnotetext{
ae-mail: reifarth@physik.uni-frankfurt.de
}

will present the capabilities of a combination of a radioactive beam facility, an ion storage ring and a high flux reactor. Such a facility would allow a direct measurement of neutron induced reactions over a wide energy range on isotopes with half lives down to minutes [14].

\section{Astrophysical Scenarios}

\section{$2.1 p$ process}

The most intensively studied astrophysical site of the $p$ process is the O-Ne layer of a massive star passed by the shock wave of a type II supernova [15]. Here, the $p$ nuclei are produced in a series of photodisintegration reactions, such as $(\gamma, \mathrm{n}),(\gamma, \mathrm{p})$, and $(\gamma, \alpha)$ reactions, from a given seed abundance. This picture is often referred to as $\gamma$ process [8]. The reaction network to describe this scenario includes a huge number of reaction rates, which are generally calculated in the framework of the Hauser-Feshbach statistical model.

An alternative model are supernovae Ia, which are associated with thermonuclear explosions of white dwarfs. An often proposed scenario is an explosion triggered once the star approaches the Chandrasekhar mass because of mass accretion from a companion main-sequence star. This is referred to as the single-degenerate scenario [16].

Figures 2 and 3 show the results of nucleosynthesis calculations following tracer particles during a supernova type Ia explosion $[17,18]$. The production and destruction 


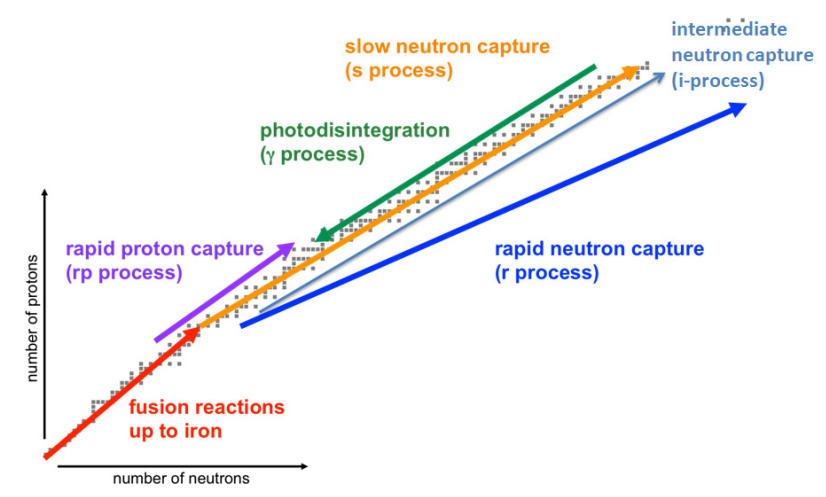

Figure 1. The $s$ and $r$ processes start with the iron peak nuclei as seeds. The s process path follows the nuclear valley of stability until it terminates in the lead-bismuth region. The $\mathrm{r}$ process drives the nuclear matter far to the neutron-rich side of the stability line and upwards until beta-delayed fission and neutroninduced fission occur and recycle the material back to smaller mass numbers. The reaction path of the $i$ process lays in between, since the corresponding intermediate neutron densities are higher than during the $s$ process, but still much smaller than during the $r$ process. Only a few isotopes on the proton-rich side of the valley of stability get significant contributions from the different models of the $p$ process.

mechanisms for the neutron-deficient isotopes ${ }^{121,123}$ I have been investigated within the framework of the Nucleosynthesis Grid (NuGrid) research platform [19]. Under the conditions investigated, the neutron evaporation process stalls at those isotopes leading to a $(\mathrm{n}, \gamma)-(\gamma, \mathrm{n})$ equilibrium as already suggested by [8]. This means, neutron capture cross section for neutron-deficient isotopes are important for the understanding of the $\gamma$ process.

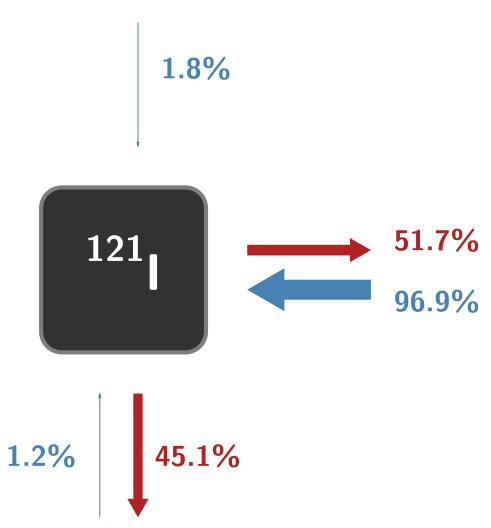

Figure 2. Reaction flows for ${ }^{121} \mathrm{I}$ during the $\gamma$ process. Blue arrows correspond to production and red arrows correspond to destruction paths. The most important production channel is ${ }^{122} \mathrm{I}(\gamma, \mathrm{n})$, while the destruction is almost equally shared between ${ }^{121} \mathrm{I}(\gamma, \mathrm{p})$ and ${ }^{121} \mathrm{I}(\mathrm{n}, \gamma)$. Neutron capture and proton removal are competing with each other.

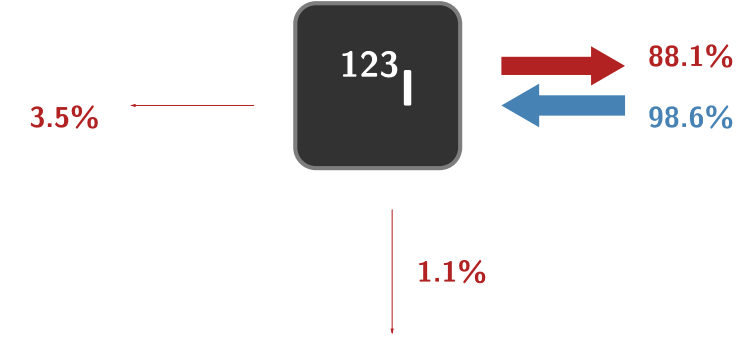

Figure 3. Reaction flows for ${ }^{123}$ I during the $\gamma$ process. Blue arrows correspond to production and red arrows correspond to destruction paths. The most important production channel is ${ }^{124} \mathrm{I}(\gamma, \mathrm{n})$, and the destruction is dominated by ${ }^{123} \mathrm{I}(\mathrm{n}, \gamma)$. The $(\mathrm{n}, \gamma)-$ $(\gamma, \mathrm{n})$ equilibrium is established.

\section{2 s process}

The modern picture of the main $s$-process component refers to the He shell burning phase in AGB stars [20]. Nuclei with masses between 90 and 209 are mainly produced during the main component. The highest neutron densities in this model occur during the ${ }^{22} \mathrm{Ne}(\alpha, \mathrm{n})$ phase and are up to $10^{12} \mathrm{~cm}^{-3}$ with temperatures around $k T=30 \mathrm{keV}$. The other extreme can be found during the ${ }^{13} \mathrm{C}(\alpha, \mathrm{n})$ phase where neutron densities as low as $10^{7} \mathrm{~cm}^{-3}$ and temperatures around $k T=5 \mathrm{keV}$ are possible. Similarly to the main component, also the weak component referring to different evolutionary stages in massive stars has two phases [21, 22]. Nuclei with masses between 56 and 90 are mainly produced during the weak component. The first phase occurs during the helium core burning with neutron densities down to $10^{6} \mathrm{~cm}^{-3}$ and temperatures around $k T=25 \mathrm{keV}$. The second phase happens during the carbon shell burning with neutron densities up to $10^{12} \mathrm{~cm}^{-3}$ at temperatures around $k T=90 \mathrm{keV}$.

Figure 4 shows the nucleosynthesis path of the $s$ process in the region around iodine. The neutron capture cross sections of ${ }^{127,129,130,131}$ I are important in order to understand the nucleosynthesis of the xenon isotopes in general and in particular the $s$-only isotopes ${ }^{128,130} \mathrm{Xe}[23]$.

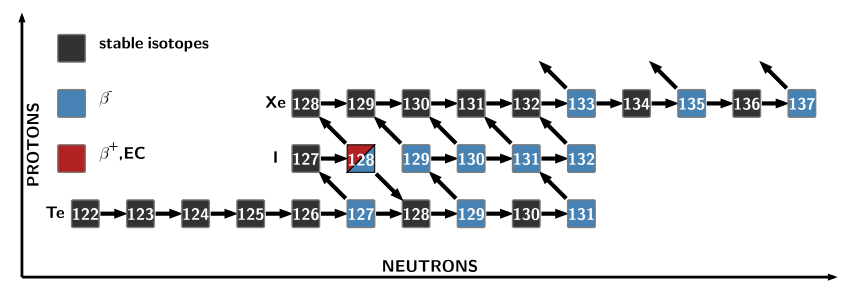

Figure 4. The s-process nucleosynthesis network around the stable ${ }^{127} \mathrm{I}$ 


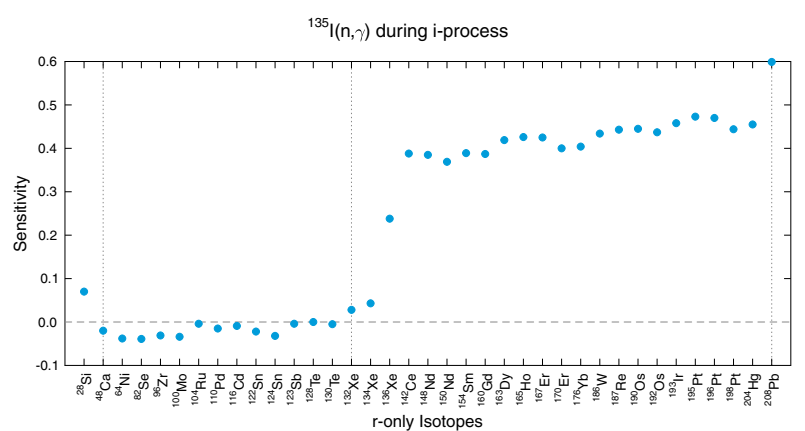

Figure 5. Impact of the ${ }^{135} \mathrm{I}(\mathrm{n}, \gamma)$ rate on the final abundances of the i process. This reaction rate affects most of the abundances beyond ${ }^{135} \mathrm{I}$ and is therefore of global importance. The sensitivity is defined as the ratio between the relative change in abundance and the relative change of the rate.

\section{3 i process}

Under certain conditions, stars may experience convective-reactive nucleosynthesis episodes. It has been shown with hydrodynamic simulations that neutron densities in excess of $10^{15} \mathrm{~cm}^{-3}$ can be reached $[4,5]$, if unprocessed, H-rich material is convectively mixed with an He-burning zone. Under such conditions, which are between the $s$ and $r$ process, the reaction flow occurs a few mass units away from the valley of stability. These conditions are sometimes referred to as the i process (intermediate process). One of the most important rates, but extremely difficult to determine, is the neutron capture on ${ }^{135} \mathrm{I}$, Figure 5. The half-life of ${ }^{135} \mathrm{I}$ is about $6 \mathrm{~h}$. Therefore, the ${ }^{135} \mathrm{I}(\mathrm{n}, \gamma)$ cross section cannot be measured directly with current facilities.

\section{Neutron capture measurements in inverse kinematics}

As discussed in the previous section on the example of the isotopic chain of iodine, the astrophysical interesting neutron capture rates range from the neutron-deficient ${ }^{121} \mathrm{I}$ $\left(t_{1 / 2}=2.1 \mathrm{~h}\right)$ to the neutron-rich ${ }^{135} \mathrm{I}\left(t_{1 / 2}=6.0 \mathrm{~h}\right)$ and more long-lived isotopes in between. Ion storage rings turned out to be powerful tools for the investigation of charged-particle-induced reactions in inverse kinematics $[24,25]$. The major advantage is the possibility of effectively shooting the ions through a thick target by passing a thin target multiple times. Since the corresponding cross sections are dominated by the tunneling probability through the Coulomb barrier, they show a very strong energy dependence. It is therefore desirable to re-accelerate the ions after each pass, which is possible using electron coolers.

This idea can be taken a step further by considering a neutron target. Since neutrons are unstable, they will have to be constantly produced. This can be done very efficiently using a reactor. The beam pipe containing the revolving ions has to go through the neutron field, which means, either through or at least close by the reactor core, see Figure 6.

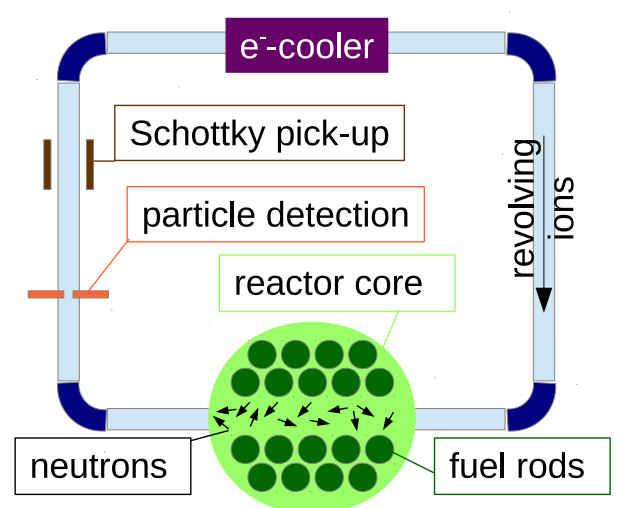

Figure 6. Schematic drawing of the proposed setup. Shown are: The main components of an ion storage ring which include the beam lines and focusing elements (blue), dipoles (dark blue), electron cooler (purple), an intersected reactor core (green), particle detection capability (orange) and Schottky pick-up electrodes (brown) [14].

In order to discuss the possible reactions, which could be investigated with this setup, it is important to understand the kinematics. The radius $(r)$ of a trajectory of a charged $(q)$ massive $(m)$ particle with velocity $(v)$ in a homogeneous, perpendicular magnetic field $(B)$ follows immediately from the Lorentz force:

$$
r=\frac{m v}{q B}=\frac{p}{q B}
$$

Equation 1 is even valid for relativistically moving particles, if $p$ and $m$ are relativistic variables. Compared to the revolving beam energy (energies above $0.1 \mathrm{AMeV}$ ), the neutrons (energies of $25 \mathrm{meV}$ ) can always be considered to be at rest. If one assumes that all channels can be viewed as a compound reaction, first a nucleus $X+n$ is formed and in a second step, particles or photons are emitted. This means, the momentum and the charge of the revolving unreacted beam $X$ and the compound nucleus $X+n$ are the same, hence both species will be on the same trajectory. However, the velocity, hence the revolution frequency, is reduced by the factor $A /(A+1)$. If the revolving ions have charge $Z=q / e$ and mass $A=12 \cdot \mathrm{m} / \mathrm{m}_{12} \mathrm{C}$, one finds then for the ratio of radii:

$$
\frac{r_{D}}{r_{P}}=\frac{Z_{P}}{Z_{D}} \frac{p_{D}}{p_{P}}=\frac{Z_{P}}{Z_{D}} \frac{A_{P}}{A_{P}+1} \frac{A_{D}}{A_{P}},
$$

where indices $D$ and $P$ denote the produced daughter and unreacted parent nuclei, respectively. And finally one obtains:

$$
\frac{r_{D}}{r_{P}}=\frac{Z_{P}}{Z_{D}} \frac{A_{D}}{A_{P}+1}
$$

It is important to note, that the underlying assumption for these simple equations is that the Q-value of the 
reaction can be neglected compared to the energy of the revolving ions. If this assumption is not valid anymore, the trajectories of the products will scatter. This holds true in particular for (exothermal) fission reactions, where the Q-value is in the order of $1 \mathrm{AMeV}$, Figure 7. Therefore the detection mechanisms described below will only be possible for fission, if the beam energy is higher than $\approx 10 \mathrm{AMeV}$. This corresponds to neutron-induced fission cross section for neutron energies of $10 \mathrm{MeV}$ or higher.
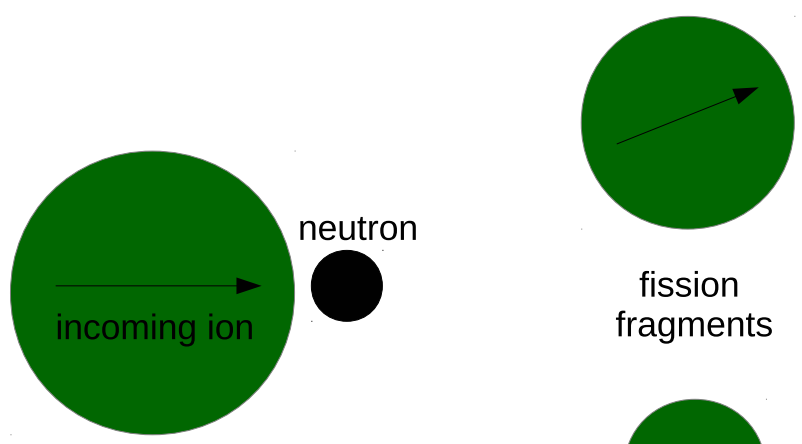

fission fragments

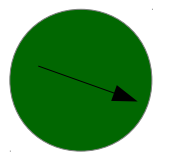

Figure 7. Because of the huge amount of energy released during a fission event, the fission products will only stay in a forward cone, if the beam energy is above $E \approx 10 \mathrm{AMeV}$.

If the magnetic rigidities of the reaction products and the primary beam are different, the reaction products can be detected using particle detectors placed in the ultrahigh ring vacuum. This is for instance possible with double sided silicon strip detectors on ceramic carriers. The position and energy deposition in the detector allows the distinction between the reaction products of interest and background [24]. According to Eq. (3) this holds true for $(\mathrm{n}, \alpha),(\mathrm{n}, \mathrm{p}),(\mathrm{n}, 2 \mathrm{n})$ and $(\mathrm{n}, \mathrm{f})$ reactions.

Neutron capture reactions have to be treated differently, since projectile (primary beam) and product will have the same trajectory, Eq. (3). In combination with an electron cooler, the number of daughter ions can then be monitored by a non-destructive Schottky spectroscopy [26, 27] or by using a sensitive SQUID-based CCC-detectors [28]. It has been shown that even single ions can be detected, even if the primary beam is still present in the ring [29].

Storage rings can be moved. Currently there are detailed plans, published as a technical design report, to move the Test Storage Ring (TSR), which was in operation until 2013 at the Max-Planck Institute for Nuclear Physics in Heidelberg to CERN where it shall be coupled to the ISOLDE radioactive ion beam facility [30].

An ISOL-type radioactive ion beam facility could be the source of the exotic nuclei. ISOL-beams combine high intensity and good quality [31]. For this particular application, the charge state of the revolving ions is not important, except for the constraints concerning the difference in trajectory with charged particles in the exit channel.

\section{Acknowledgements}

This project was supported by the Helmholtz International Center for FAIR, the Helmholtz-CAS Joint Research Group HCJRG108, the GIF project G-1051-103.7/2009, the BMBF project 05P12RFFN6 and the EuroGenesis project MASCHE. The numerical calculations for tracer particles in SNIa have been supported by the B2Fh Association and the Goethe University Frankfurt.

\section{References}

[1] E. Anders, N. Grevesse, Geochim. Cosmochim. Acta 53, 197 (1989)

[2] F.K. Thielemann, C. Fröhlich, R. Hirschi, M. Liebendörfer, I. Dillmann, D. Mocelj, T. Rauscher, G. Martinez-Pinedo, K. Langanke, K. Farouqi et al., Progress in Particle and Nuclear Physics 59, 74 (2007)

[3] R. Reifarth, C. Lederer, F. Käppeler, Journal of Physics G Nuclear Physics 41, 053101 (2014)

[4] F. Herwig, M. Pignatari, P.R. Woodward, D.H. Porter, G. Rockefeller, C.L. Fryer, M. Bennett, R. Hirschi, Ap. J. 727, 89 (2011)

[5] D.A. García-Hernández, O. Zamora, A. Yagüe, S. Uttenthaler, A.I. Karakas, M. Lugaro, P. Ventura, D.L. Lambert, A\&A. 555, L3 (2013)

[6] K. Lodders, Meteoritics and Planetary Science Supplement 38, 5272 (2003)

[7] F.K. Thielemann, D. Mocelj, I. Panov, E. Kolbe, T. Rauscher, K.L. Kratz, K. Farouqi, B. Pfeiffer, G. Martinez-Pinedo, A. Kelic et al., International Journal of Modern Physics E 16, 1149 (2007)

[8] M. Arnould, S. Goriely, Physics Reports 384, 1 (2003)

[9] F. Käppeler, R. Gallino, S. Bisterzo, W. Aoki, Reviews of Modern Physics 83, 157 (2011)

[10] C. Langer, O. Lepyoshkina, Y. Aksyutina, T. Aumann, S.B. Novo, J. Benlliure, K. Boretzky, M. Chartier, D. Cortina, U.D. Pramanik et al., Phys. Rev. C 89, 035806 (2014)

[11] S.G. Altstadt, T. Adachi, Y. Aksyutina, J. Alcantara, H. Alvarez-Pol, N. Ashwood, L. Atar, T. Aumann, V. Avdeichikov, M. Barr et al., Nuclear Data Sheets 120, 197 (2014)

[12] K. Göbel, P. Adrich, S. Altstadt, H. Alvarez-Pol, F. Aksouh, T. Aumann, M. Babilon, K. Behr, J. Benlliure, T. Berg et al., Journal of Physics Conference Series in press (2014)

[13] M.B. Chadwick, M. Herman, P. Obložinský, M.E. Dunn, Y. Danon, A.C. Kahler, D.L. Smith, B. Pritychenko, G. Arbanas, R. Arcilla et al., Nuclear Data Sheets 112, 2887 (2011)

[14] R. Reifarth, Y.A. Litvinov, Phys. Rev. ST Accel. Beams 17, 014701 (2014)

[15] T. Hayakawa, N. Iwamoto, T. Kajino, T. Shizuma, H. Umeda, K. Nomoto, Ap. J. 685, 1089 (2008)

[16] W. Hillebrandt, J.C. Niemeyer, Annual Review of Astronomy and Astrophysics 38, 191 (2000) 
[17] C. Travaglio, F.K. Röpke, R. Gallino, W. Hillebrandt, Ap. J. 739, 93 (2011)

[18] S. Bisterzo, C. Travaglio, R. Gallino, M. Wiescher, F. Käppeler, Ap. J. 787, 10 (2014)

[19] M. Pignatari, F. Herwig, Nuclear Physics News 22, 18 (2012)

[20] M. Lugaro, F. Herwig, J.C. Lattanzio, R. Gallino, O. Straniero, Ap. J. 586, 1305 (2003)

[21] C. Travaglio, R. Gallino, E. Arnone, J. Cowan, F. Jordan, C. Sneden, Ap. J. 601, 864 (2004)

[22] M. Heil, F. Käppeler, E. Uberseder, R. Gallino, M. Pignatari, Prog. Nucl. Part. Phys. 59, 174 (2007)

[23] R. Reifarth, F. Käppeler, F. Voss, K. Wisshak, R. Gallino, M. Pignatari, O. Straniero, Ap. J. 614, 363 (2004)

[24] Q. Zhong, T. Aumann, S. Bishop, K. Blaum, K. Boretzky, F. Bosch, H. Bräuning, C. Brandau, T. Davinson, I. Dillmann et al., Journal of Physics: Conference Series 202, 012011 (2010)

[25] Y.A. Litvinov, S. Bishop, K. Blaum, F. Bosch, C. Brandau, L.X. Chen, I. Dillmann, P. Egelhof, H. Geissel, R.E. Grisenti et al., Nuclear Instruments and Methods in Physics Research B 317, 603 (2013)

[26] M. Grieser, R. Bastert, K. Blaum, H. Buhr, D. Fischer, F. Laux, R. Repnow, T. Sieber, A. Wolf, R. von
Hahn et al., The Diagnostics System at the Cryogenic Storage Ring CSR, in IPAC2010 (Kyoto, Japan, http://accelconf.web.cern.ch/AccelConf/IPAC10/, 2010), Vol. MOPD092, p. 918

[27] F. Nolden, P. Hülsmann, Y. Litvinov, P. Moritz, C. Peschke, P. Petri, M. Sanjari, M. Steck, H. Weick, J. Wu et al., Nucl. Instr. Meth A 659, 69 (2011)

[28] W. Vodel, R. Geithner, R. Neubert, P. Seidel, K.K. Knaack, K. Wittenburg, A. Peters, H. Reeg, M. Schwickert, 20 Years of Development of SQUID-based Cryogenic Current Comparator for Beam Diagnostics, in IPAC2013 (Shanghai, China, http://accelconf.web.cern.ch/accelconf/IPAC2013/, 2013), Vol. MOPME013, p. 497

[29] D. Shubina, R.B. Cakirli, Y.A. Litvinov, K. Blaum, C. Brandau, F. Bosch, J.J. Carroll, R.F. Casten, D.M. Cullen, I.J. Cullen et al., Phys. Rev. C 88, 024310 (2013)

[30] M. Grieser, Y.A. Litvinov, R. Raabe, K. Blaum, Y. Blumenfeld, P.A. Butler, F. Wenander, P.J. Woods, M. Aliotta, A. Andreyev et al., European Physical Journal Special Topics 207, 1 (2012)

[31] K.H. Schmidt, A. Kelić, S. Lukić, M.V. Ricciardi, M. Veselsky, Phys. Rev. ST Accel. Beams 10, 014701 (2007) 
